\title{
Expression and clinical significance of Sirt1 in colorectal cancer
}

\author{
DENG-FENG YU ${ }^{1,2}$, SU-JUAN JIANG ${ }^{1,3}$, ZHI-PENG PAN $^{1}$, WEI-DONG CHENG ${ }^{2}$, WEN-JUN ZHANG ${ }^{2}$, \\ XIAO-KUN YAO ${ }^{1}$, YU-CHENG $\mathrm{LI}^{4}$ and YONG-ZHI LUN ${ }^{1}$
}

\author{
${ }^{1}$ Liaoning Provincial University Key Laboratory of Biophysics, College of Medicine, Dalian University, Dalian, \\ Liaoning 116622; ${ }^{2}$ Department of Anorectal Surgery, Affiliated Xinhua Hospital of Dalian University, Dalian, \\ Liaoning 116021; ${ }^{3}$ Department of Gynecology and Obstetrics, Affiliated Zhongshan Hospital of Dalian University, Dalian, \\ Liaoning 116001; ${ }^{4}$ Department of Dermatology, Yuzhou People's Hospital, Xuchang, Henan 461670, P.R. China
}

Received January 23, 2015; Accepted October 6, 2015

DOI: $10.3892 / 01.2015 .3982$

\begin{abstract}
The objective of the present study was to examine the expression of Silent information regulator 1 (Sirt1) in colorectal cancer and peritumoral normal mucosa tissue, and therefore analyze the role and molecular mechanism of Sirt1 in the pathogenesis of colorectal cancer. Colorectal cancer tissue specimens were employed as the experimental group, and adjacent normal mucosa tissues $>5 \mathrm{~cm}$ from tumor lesions were used as the control group. The expression of Sirt1 was detected by the immunohistochemical streptavidin peroxidase detection method in paraffin-embedded sections, whilst Sirt1 protein expression was examined by western blot analysis in the fresh tissues. Sirt1 protein was primarily expressed in the nuclei of the tumor cells, and positive staining was brownish-yellow in color. The relative expression quantities of Sirt1 in the peritumoral normal rectal mucosa and rectal carcinoma were 1.15 and 2.62 , and the differences between the two groups were statistically significant $(\mathrm{P}<0.05)$. The expression level of Sirt1 in colorectal carcinoma was significantly associated with the depth of tumor invasion, differentiation and tumor size $(\mathrm{P}<0.05)$. Sirtl expression was also found to be associated with tumor tissue type, lymph node metastasis, Duke's stage and patient age. These characteristics combined may therefore be used as markers for the early diagnosis of colorectal cancer pathogenesis.
\end{abstract}

\section{Introduction}

Colorectal cancer occurs in the colorectal mucosa and colonic glands and is one of the most common types of malignant tumor of the digestive system $(1,2)$. The worldwide incidence and mortality rates of colorectal cancer increased significantly

Correspondence to: Dr Yong-Zhi Lun, Liaoning Provincial University Key Laboratory of Biophysics, College of Medicine, Dalian University, 10 Xuefu Street, Dalian, Liaoning 116622, P.R. China

E-mail: lunyz@163.com

Key words: Sirt1, colorectal cancer, expression, clinical significance between 2009 and 2013; in 2013, a total of 142,820 new cases were diagnosed and 50,830 mortalities occurred as a result of colorectal cancer (3). Thus, this malignancy presents a serious threat to human health $(1,2)$. Clinical and pathological data of patients with colorectal cancer in China have demonstrated the following pathogenic characteristics: The number of patients in cities is higher than that in the countryside, indicating a clear urbanization trend; and patients $<30$ years of age account for $>10 \%$ of the total number of patients, demonstrating a clear trend towards older age (4). Surgical resection is currently the main method used for the treatment of colorectal cancer. However, as significant symptoms are often not present in the early stages of the disease, patients are frequently diagnosed in the later stages; by this time, the optimal period for surgery has passed. Furthermore, metastasis or recurrence occurs in a large number of patients following surgical resection, affecting the prognosis of the patients. Therefore, the five-year survival rate is low $(64 \%)$, posing a serious threat to patient health $(5,6)$.

The occurrence and pathogenesis of colorectal cancer is a complex, multistep process, regulated by a number of different genes (1). A total of $25 \%$ of patients with colorectal cancer have a genetic history, which is associated with familial adenomatous polyposis and hereditary non-polyposis colorectal cancer (7). Results of molecular pathology and colorectal cancer expression profile chip screening have demonstrated that multiple genes serve regulatory roles in the processes of colorectal cancer development (8). In order to identify possible gene target therapies and individualized treatments, various studies have focused on genes associated with the pathogenesis of colorectal cancer and their mechanisms. Previous studies have employed various methods to explore potential markers for the early diagnosis and prognosis of colorectal cancer; however, at present, its primary cause is unknown, and therefore further research is required (9).

Recent studies have demonstrated that Silent information regulator 1 (Sirt1) can regulate the deacetylation of lysine residues of multiple proteins, which is dependent upon nicotinamide adenine dinucleotide $\left(\mathrm{NAD}^{+}\right)$. Sirt1, a member of the Sirtuin family, is a type of class III histone deacetylase (10). The Sirtuin family regulates gene expression and is involved in the regulation of various biological events in cells. In particular, Sirtl is important in cell survival, senescence, apoptosis, differentiation and other metabolic processes (11). Studies 
have demonstrated that the expression of Sirt1 is increased in prostate cancer (12) and acute myelocytic leukemia (13). Hida et al (14) observed that the expression of Sirt1 was also significantly enhanced in a variety of types of non-melanoma skin cancer, including squamous cell carcinoma, basal cell carcinoma, Bowen's disease and actinic keratosis. The $\mathrm{NAD}^{+}$-dependent deacetylase, Sirt1, is involved in cellular survival pathways, which ensure that the tumor suppressor gene, p53, and members of the forkhead transcription factor family remain deacetylated (15). Therefore, Sirt1 is considered to promote cancer genes and it may be involved in the regulation of tumor formation and initiation and developmental processes (15).

In order to further explore the significance and mechanism of Sirt1 in the pathogenesis of colorectal carcinoma, the present study investigated the expression of Sirt1 in colorectal carcinoma tissues and normal colorectal mucosa by means of the immunohistochemistry streptavidin peroxidase (SP) method and western blot analysis, and analyzed the associations between the expression levels of Sirt1 and clinicopathological factors. The results may provide novel ideas and inspiration for the research of colorectal cancer etiology.

\section{Materials and methods}

Research subjects. The present study was approved by the Affiliated Xinhua Hospital of Dalian University (Dalian, China) ethics committee. Specimens were collected with informed consent, and the investigation did not affect the disease diagnosis or follow-up treatment of the patients. Specimens from 40 patients undergoing surgical resection for the treatment of colorectal cancer were collected between March 2010 and October 2012 at the Affiliated Xinhua Hospital of Dalian University. The colorectal cancer tissue specimens represented the experimental group, and the adjacent normal mucosa tissues ( $>5 \mathrm{~cm}$ from tumor lesions) represented the control group. The patients included in the study had not previously received surgery for colorectal cancer, and did not exhibit endocrine or immune system disease. Patients who had received chemotherapy were excluded from the study. Hormonal treatment was also not conducted within three months prior to the surgery. Samples were acquired from the specimens within $30 \mathrm{~min}$ of surgical removal, and each sample was divided into two. One part of the sample was fixed in $10 \%$ formalin for the preparation of the paraffin specimens, and the expression of Sirt1 was examined in these samples by the immunohistochemical SP method. The second part of the sample was stored in liquid nitrogen, and the expression of Sirtl protein in the fresh tissues was detected by western blot analysis. The clinical and pathological data characteristics of the patients were assessed, and the association between the expression of Sirt1 and the clinical pathological data was analyzed. The experimental group and the control group included a total of 80 samples from 40 patients, comprising 27 males and 13 females with a mean age of $59.37 \pm 10.05$ years. Hematoxylin and eosin staining identified 12 cases of colon cancer and 28 cases of rectal cancer in the experimental group, whilst the control group samples were composed of normal mucosa. The 40 cases of colorectal cancer consisted of 22 cases of predominantly ulcerative type and 18 cases of exophytic type tumors, with no cases of polypoid type tumors (16). According to histological grading (17), there were 15 cases of high differentiation, 19 cases of moderate differentiation and 6 cases of low differentiation. With regard to lymph node metastasis, there were 19 cases without lymph node metastasis and 21 cases with lymph node metastasis. According to Duke's classification (18), there were 18 cases of stage $\mathrm{A}+\mathrm{B}$ and 22 cases of stage $\mathrm{C}+\mathrm{D}$.

Main reagents. The Sirt1 rabbit anti-human polyclonal antibody (cat. no. sc-15404) was purchased from Santa Cruz Biotechnology, Inc. (Dallas, TX, USA). The universal immunohistochemical Streptavidin-Peroxidase staining kit (cat. no. SP-9000), diaminobenzidine (DAB) developing liquid and mouse anti-human GAPDH monoclonal antibody (cat. no. TA-08) were purchased from Beijing Zhongshan Jinqiao Biotechnology Co., Ltd. (Beijing, China). RIPA strong lysis buffer, the protease inhibitor phenylmethanesulfonyl fluoride (PMSF), a BCA Protein Assay kit and a Beyo ECL Plus kit were purchased from Beyotime Institute of Biotechnology (Nanjing, China).

Immunohistochemical staining of paraffin sections using the SP method. Paraffin embedding and sectioning of colorectal cancer and normal mucosa were conducted. Goat serum blocking solution (Beijing Zhongshan Jinqiao Biotechnology Co., Ltd.) was applied dropwise at room temperature for 15 min. The Sirt1 primary antibody (1:100) was added and incubated with samples at $4^{\circ} \mathrm{C}$ overnight. A horseradish peroxidase-conjugated goat anti-mouse IgG secondary antibody (cat. no. ZDR-5307; 1:300; Beijing Zhongshan Jinqiao Biotechnology Co., Ltd.) was added to the samples and incubated at room temperature for $30 \mathrm{~min}$. Each $4-\mu \mathrm{m}$ section was treated with $50 \mu \mathrm{l}$ horseradish peroxidase-labeled streptavidin working fluid (Beijing Zhongshan Jinqiao Biotechnology Co., Ltd.). After the DAB liquid had developed for $3 \mathrm{~min}$, the nuclei were counterstained with hematoxylin (Beijing Zhongshan Jinqiao Biotechnology Co., Ltd.) for $40 \mathrm{sec}$, followed by dehydration. The dehydrated sections were cover-slipped with neutral gum for microscopic examination (Axiolab; Carl Zeiss AG, Oberkochen, Germany). The positive staining graph included with the kit served as the positive control, and phosphate-buffered saline replaced Sirt1 and served as the negative control.

Photographs were captured from 5 arbitrary fields in each section using a digital camera (Nikon COOLPIX S9500; Nikon Corporation, Tokyo, Japan), and the double-blind method was used for the data statistics. The percentage of positively stained cells from the total cells in each field was scored as follows: <1\%, 0 points; 1-20\%, 1 point; 21-50\%, 2 points; and $>50 \%, 3$ points. The positive staining intensity was then scored as follows: No coloring, 0 points; pale yellow, 1 point; brown-yellow, 2 points; and sepia, 3 points. The product of these two scores served as the overall section staining score.

Western blot analysis. Tissue proteins were extracted using the RIPA strong lysis buffer and PMSF. The concentration of protein was measured according to the instructions of the BCA kit. The protein samples were analyzed by $10 \%$ SDS-PAGE. After transferring the samples to a polyvinylidene fluoride 
Table I. Sirt1 staining score as detected by the immunohistochemical streptavidin peroxidase method.

\begin{tabular}{lcc}
\hline Group & Cases & $\begin{array}{c}\text { Sirt1 staining } \\
\text { score }\end{array}$ \\
\hline Normal tissues & 40 & $1.15 \pm 0.419$ \\
Cancer tissues & 40 & $2.62 \pm 0.537^{\mathrm{a}}$ \\
\hline
\end{tabular}

Staining score data are presented as the mean \pm standard deviation. ${ }^{\mathrm{a}} \mathrm{P}<0.05$ vs. normal tissues. Sirt1, Silent information regulator 1 .

Table II. Association between Sirt1 expression and clinicopathological data of patients with colorectal cancer.

\begin{tabular}{|c|c|c|c|}
\hline Variable & Cases & $\begin{array}{l}\text { Sirt1 staining } \\
\text { score }\end{array}$ & P-value \\
\hline Gender & & & 0.084 \\
\hline Male & 27 & $2.35 \pm 0.347$ & \\
\hline Female & 13 & $2.89 \pm 0.561$ & \\
\hline Age, years & & & $<0.0001$ \\
\hline$<50$ & 16 & $1.64 \pm 0.401$ & \\
\hline$\geq 50$ & 24 & $2.91 \pm 0.514$ & \\
\hline Morphological type & & & $<0.0001$ \\
\hline Ulcerative & 22 & $1.58 \pm 0.462$ & \\
\hline Exophytic & 18 & $2.93 \pm 0.618$ & \\
\hline Tissue differentiation & & & $<0.0001$ \\
\hline High & 15 & $1.46 \pm 0.471$ & \\
\hline Medium or low & 25 & $2.79 \pm 0.630$ & \\
\hline Depth of invasion & & & $<0.0001$ \\
\hline Shallow muscle layer & 19 & $1.60 \pm 0.513$ & \\
\hline Whole layer & 21 & $2.97 \pm 0.437$ & \\
\hline Lymph node metastasis & & & $<0.0001$ \\
\hline Yes & 21 & $1.53 \pm 0.428$ & \\
\hline No & 19 & $2.95 \pm 0.643$ & \\
\hline Duke's stage & & & $<0.0001$ \\
\hline $\mathrm{A}+\mathrm{B}$ & 19 & $1.49 \pm 0.501$ & \\
\hline $\mathrm{C}+\mathrm{D}$ & 21 & $2.92 \pm 0.713$ & \\
\hline
\end{tabular}

Sirt1 staining score data are presented as the mean \pm standard deviation. Sirt1, Silent information regulator 1 .

membrane (Beyotime Institute of Biotechnology), 5\% skim milk powder was applied for blocking and samples were incubated at $4^{\circ} \mathrm{C}$ overnight. After washing the membrane with Tris-Buffered saline with Tween 20, the protein samples were incubated with the polyclonal rabbit anti-human polyclonal Sirt1 (cat. no. sc-15404; Santa Cruz Biotechnology, Inc.) and monoclonal mouse anti-human GAPDH antibodies (cat. no. TA-08; Beijing Zhongshan Jinqiao Biotechnology Co., Ltd.) at $4^{\circ} \mathrm{C}$ overnight, followed by the horseradish peroxidase-conjugated goat anti-mouse IgG secondary antibody (cat. no. ZDR-5307; Beijing Zhongshan Jinqiao Biotechnology
Table III. Gray value of the Sirt1 protein as detected by western blot analysis.

\begin{tabular}{lcc}
\hline Group & Cases & Sirt1/GAPDH gray value \\
\hline Normal tissues & 40 & $0.72 \pm 0.327$ \\
Tumor tissues & 40 & $1.21 \pm 0.245^{\mathrm{a}}$ \\
\hline
\end{tabular}

Sirt1/GAPDH gray value data are presented as the mean \pm standard deviation. ${ }^{\text {a }}<<0.05$ vs. normal tissues. Sirt1, Silent information regulator 1 .

Co., Ltd.) at $4^{\circ} \mathrm{C}$ overnight. Subsequently, the membranes were placed in a chemiluminescence imaging instrument (ImageQuant LAS 4000 Mini; GE Healthcare Bio-Sciences, Pittsburgh, PA, USA), then exposure and image capture and analyses were conducted (ImageQuant TL 1.0 software; GE Healthcare Bio-Sciences).

Statistical analysis. SPSS software version 22.0 (IBM SPSS, Armonk, NY, USA) was used for statistical analysis. The data are expressed as the mean \pm standard deviation. A paired-samples t-test was employed to analyze the expression of Sirtl in the colorectal cancer tissues and the normal mucosa tissues. One-way analysis of variance was performed to analyze the differences in Sirt1 expression in the tissues associated with the various clinical and pathological variables. $\mathrm{P}<0.05$ was considered to indicate a statistically significant difference.

\section{Results}

Expression levels of Sirt1 in paraffin-embedded colorectal cancer and normal mucosa samples detected by immunohistochemistry. As indicated by the arrows in Fig. 1, Sirt1 was primarily expressed in the nucleus, with stained nuclei indicating positive staining. As illustrated in Fig. 1B, Sirt1 was highly expressed in the colorectal cancer samples; the area of positive staining was large. By contrast, the expression of Sirt1 was weaker and the area of positive staining was more limited in normal mucosa samples (Fig. 1A).

The expression levels of Sirt1 in cancer tissues and normal mucosa were analyzed according to the evaluation standards. Multiplication of the staining percentage and staining intensity scores were conducted to calculate the mean overall section staining values of the samples in the experimental and control groups. A significant difference was identified between the two groups $(\mathrm{P}<0.05$; Table I).

The associations between Sirt1 expression and clinicopathological variables are presented in Table II. The mean staining score for Sirt1 was $2.35 \pm 0.347$ ( \pm standard deviation) and $2.89 \pm 0.561$ in male and female patients, respectively. However, no significant differences in Sirt1 expression were identified between males and females $(\mathrm{P}=0.084)$. The mean staining score for Sirt1 was $1.64 \pm 0.401$ and $2.91 \pm 0.514$ in patients aged $<50$ and $\geq 50$ years, respectively, and this difference was determined to be statistically significant $(\mathrm{P}<0.0001)$. The mean staining score for Sirt1 in 22 patients with ulcerative type colorectal cancer was $1.58 \pm 0.462$, while in 18 patients 

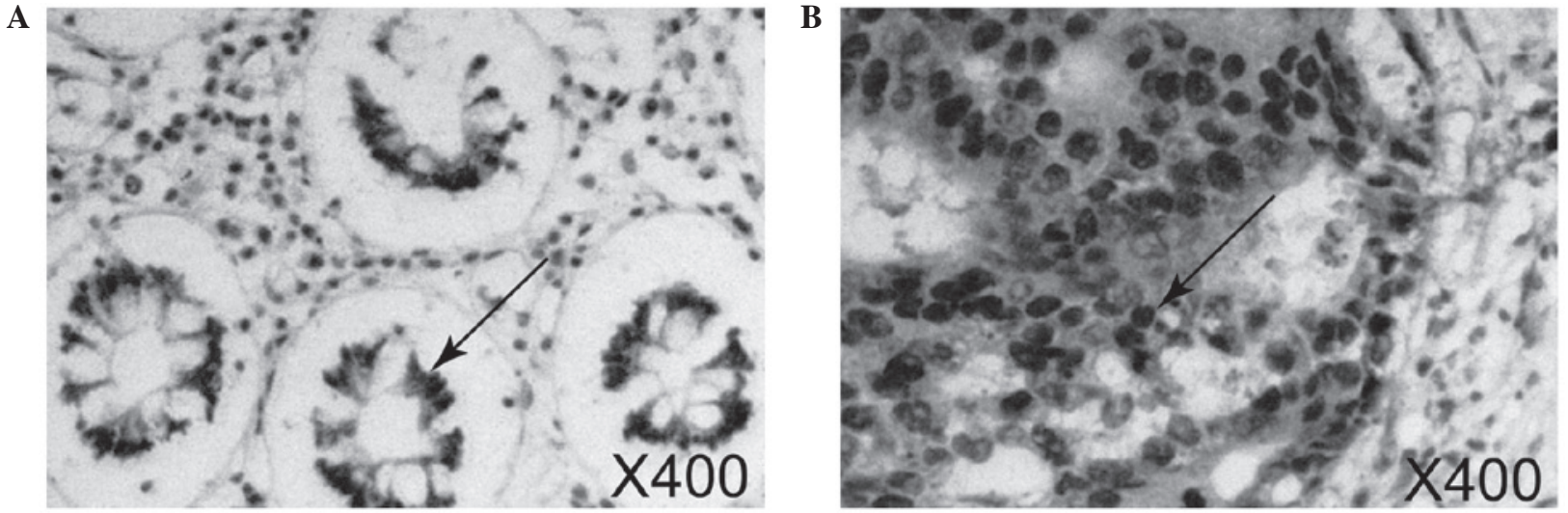

Figure 1. Immunohistochemical staining of Sirt1 in (A) normal mucosa tissue and (B) colorectal cancer tissue. Arrows indicate positive Sirt1 staining. Sirt1, Silent information regulator 1 .

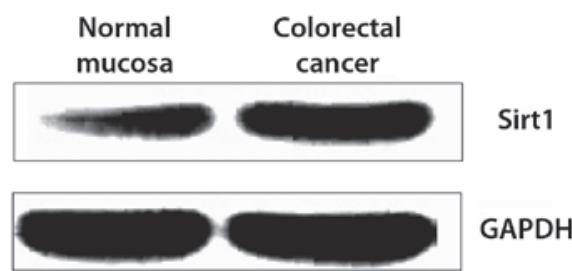

Figure 2. Expression of Sirt1 protein in colorectal carcinoma and normal mucosa tissues assessed by western blot. Sirt1, Silent information regulator 1 .

with exophytic type the mean staining score for Sirt1 was $2.93 \pm 0.618$. This difference was statistically significant $(\mathrm{P}<0.0001)$. The mean staining score for Sirt1 in 15 patients with highly differentiated cancer was $1.46 \pm 0.471$, whereas in the 25 patients exhibiting medium and low differentiation, the mean staining score was $2.79 \pm 0.630$, and this difference was found to be statistically significant $(\mathrm{P}<0.0001)$. Regarding tissue differentiation, the 19 patients with superficial muscular layer differentiation exhibited a mean Sirt1 staining score of $1.60 \pm 0.513$, whereas the 21 patients exhibiting whole layer differentiation exhibited a mean score of $2.97 \pm 0.437$, and this difference was determined to be statistically significant $(\mathrm{P}<0.0001)$. The mean staining score for Sirt1 in 21 patients with lymph node metastasis was $1.53 \pm 0.428$, and in 19 patients with lymph node metastasis the score was $2.95 \pm 0.643$. This difference was also determined to be statistically significant $(\mathrm{P}<0.0001)$. The mean Sirt1 staining score for the 19 patients with A+B Duke's stage cancer was $1.49 \pm 0.501$, whereas the mean Sirtl staining score of the 21 patients with $\mathrm{C}+\mathrm{D}$ Duke stage cancer was $2.92 \pm 0.713$, and this difference was statistically significant $(\mathrm{P}<0.0001)$. Therefore, the present results revealed that older age, lower tissue differentiation, deeper depth of invasion, lymph node metastasis and higher Duke's stage are associated with higher Sirt1 expression $(\mathrm{P}<0.0001)$. Furthermore, in terms of morphological type, Sirt1 expression is higher in exophytic compared with ulcerating tumors $(\mathrm{P}<0.0001)$; however, Sirt1 expression is not significantly associated with patient gender $(\mathrm{P}=0.084)$.

Expression of Sirt1 protein in fresh colorectal cancer and normal mucosa tissue samples detected by western blotting.
According to the detection results acquired by the SP immunohistochemistry method, Sirt1 may be an important factor in colorectal cancer, as indicated by the significantly increased expression of Sirt1 in the paraffin-embedded carcinoma specimens. To verify this observation, the expression of Sirtl protein in fresh tumor tissues and normal tissues was analyzed simultaneously by western blot analysis. Expression was analyzed in 40 tissue specimens in each group, and the expression levels of Sirt1 were presented as the gray value of Sirt1/GAPDH. The results demonstrated that Sirt1 expression in colorectal cancer tissue was elevated compared with that of normal mucosa (Fig. 2; Table III). This difference was significant $(\mathrm{P}<0.05)$.

\section{Discussion}

The occurrence and pathogenesis of colorectal cancer constitute a complex process regulated by numerous genes (1). Under the conditions of different stimuli, abnormal transcription and translation of multiple genes induces changes in the expression of signaling proteins. Subsequently, signal conduction pathways become uncontrolled, leading to an imbalance in cell growth, survival, differentiation and proliferation (19). The protein acetylation/deacetylation cycle is also important in the process of gene expression and regulation (20). Protein acetylation and deacetylation are catalyzed by histone acetyltransferases and histone deacetylases, and Sirt1 belongs to the class III histone deacetylases and is a member of the Sirtuin family. This family catalyzes the deacetylation of lysine residues on various proteins in a NAD ${ }^{+}$-dependent manner $(10,21)$.

Multiple studies have demonstrated that the possible regulatory mechanism of Sirt1 as a cancer gene is associated with tumor protein p53 (22). The p53 protein is a tumor suppressor protein that serves diverse roles in multiple physiological processes within the body. Following a decrease in its expression levels, or if gene mutation occurs, the p53 protein can no longer fulfill these roles, thus increasing the risk of cancer (23). Sirt1 may induce p53 loss-of-function through the deacetylation of p53 at the Lys382 residue, which is contained in the C-terminus, consequently losing the ability to suppress tumor formation (24). Furthermore, studies have also demonstrated that, under conditions of DNA damage and oxidative stress, the 
overexpression of Sirt1 may inhibit the p53-regulated cell cycle and lead to the arrest of cell replication and cell apoptosis (25). In addition, the deacetylase activity of Sirt1 was observed to decrease when Sirt1 mutants were transcribed and translated using the site-directed mutagenesis method in a model of carcinogenesis, which increased the sensitivity of cells to DNA damage and the oxidative stress response (26). Further studies demonstrated that the addition of a specific inhibitor of Sirt1, 5' adenosine monophosphate-activated protein kinase, into human hepatoma HepG2 and PLC/PRF/5 cells led to significant reductions in the activity and function of Sirt1, resulting in increased acetylation and transcriptional activity of the p53 protein $(27,28)$. In addition, previous studies noted that Sirt1 expression in primary colorectal carcinoma was significantly increased, suggesting that Sirt1 is key in the occurrence and development of intestinal tumors (29). In the present study it was observed that Sirt1 expression in the primary colorectal cancer tissues were all significantly increased.

There are notable effects of living habits, diet structure and geographical regions on the incidence of gastrointestinal tract cancer $(7,30)$. Therefore, in order to minimize the effect of external factors on the experimental results, the current study used colorectal cancer specimens and normal mucosa samples from the same patients as the experimental and control groups, respectively. The results demonstrated that, compared with that of normal mucosa tissues, the expression of Sirt1 in colorectal cancer tissues was significantly increased. Although gender did not affect the expression of Sirt1, significant differences in the expression of Sirt1 in colorectal specimens were observed between patients of different age groups, with significantly increased expression in patients aged $\geq 50$ years. This may be associated with the putative role of Sirt1 as the longevity gene (31). Studies have indicated that the specific activators of Sirt1 contribute to the protection of cardiovascular function and prolong life (32). Therefore, the greater the age of the patient, the higher the expression of Sirt1 may be. The increased expression of Sirtl was more evident in the process of cancer cells. Due to the fact that patients with polypoid type and adhesive type tumors were fewer in number, along with the greater difficulty of accessing the normal mucosa in such patients, the present research was conducted using tumors of ulcerative and exophytic gross morphological types. In addition, as the ulcerative type tumors with low differentiation accounted for a large proportion of the sample, the degree of malignancy was high. By contrast, the proportions of high and low differentiation in patients with the exophytic type tumor were comparable, and the degree of malignancy was low. It was observed that the expression of Sirtl increased significantly in the patients with the ulcerative type. This is consistent with the observations for degree of tissue differentiation; increased Sirtl expression was enhanced in the group of patients with medium and low differentiation. The depth of invasion, lymph node metastasis and Duke's stage are also indices reflecting the degree of cancer progression and malignancy (33). According to these three indices, it was observed that the expression of Sirt1 was elevated more significantly in cases with a higher degree of malignancy and further progression of colorectal carcinoma. Therefore, these results provide strong evidence of Sirt1 as a cancer-associated gene in colorectal cancer, laying the foundation for follow-up research.
However, opposing opinions have also been presented. One study suggested that the Sirtl protein may serve inhibitory roles in the occurrence and development processes of colon cancer (34). In addition, animal tumor models have shown that Sirt1 may function as an anti-oncogene, acting as a tumor-inhibitory factor (35). Leko et al overexpressed the Sirt1 protein in $\mathrm{APC}^{\mathrm{Min} /+}$ mice, and the risk of cancer of the colon was significantly reduced (36). The mechanism underlying this may be that the overexpressed Sirt1 acts as a deacetylase, leading to the deacetylation of $\beta$-catenin in the cytoplasms of cells, thereby resulting in its nuclear localization and loss of its normal function (37). Furthermore, the expression level of Sirt1 protein in the nucleus and its deacetylation activity have been demonstrated to be negatively correlated with the expression of $\beta$-catenin (38).

In conclusion, the association between Sirtl and the occurrence and development of cancer, and the related mechanisms, are unclear at present. The leading theory is that the activation of the Sirt1 protein can increase the risk of cancer, based on the evidence that Sirt1 can deacetylate and inactivate the tumor suppressor gene p53 (26). The present investigation provides a novel direction for investigation into the early diagnosis and targeted treatment of colorectal cancer.

\section{References}

1. Azer SA: Overview of molecular pathways in inflammatory bowel disease associated with colorectal cancer development. Eur J Gastroenterol Hepatol 25: 271-281, 2013.

2. Siegel R, Ma J, Zou Z and Jemal A: Cancer statistics, 2014. CA Cancer J Clin 64: 9-29, 2014.

3. Siegel R, Naishadham D and Jemal A: Cancer statistics, 2013. CA Cancer J Clin 63: 11-30, 2013.

4. Raskov H, Pommergaard HC, Burcharth J and Rosenberg J: Colorectal carcinogenesis - update and perspectives. World J Gastroenterol 20: 18151-18164, 2014.

5. Newman NA, Votanopoulos KL, Stewart JH, Shen P and Levine EA: Cytoreductive surgery and hyperthermic intraperitoneal chemotherapy for colorectal cancer. Minerva Chir 67: 309-318, 2012.

6. Hompes D, D'Hoore A, Van Cutsem E, Fieuws S, Ceelen W, Peeters M, Van der Speeten K, Bertrand C, Legendre H and Kerger J: The treatment of peritoneal carcinomatosis of colorectal cancer with complete cytoreductive surgery and hyperthermic intraperitoneal peroperative chemotherapy (HIPEC) with oxaliplatin: A Belgian multicentre prospective phase II clinical study. Ann Surg Oncol 19: 2186-2194, 2012.

7. Fu Z, Shrubsole MJ, Smalley WE, Wu H, Chen Z, Shyr Y, Ness RM and Zheng W: Association of meat intake and meat-derived mutagen exposure with the risk of colorectal polyps by histologic type. Cancer Prev Res (Phila) 4: 1686-1697, 2011.

8. Piepoli A, Tavano F, Copetti M, Mazza T, Palumbo O, Panza A, di Mola FF, Pazienza V, Mazzoccoli G, Biscaglia G, et al: MiRNA expression profiles identify drivers in colorectal and pancreatic cancers. PLoS One 7: e33663, 2012.

9. Linnekamp JF, Wang X, Medema JP and Vermeulen L: Colorectal cancer heterogeneity and targeted therapy: A case for molecular disease subtypes. Cancer Res 75: 245-249, 2015.

10. Blander $G$ and Guarente L: The Sir2 family of protein deacetylases. Annu Rev Biochem 73: 417-435, 2004.

11. Casatta N, Porro A, Orlandi I, Brambilla L and Vai M: Lack of Sir2 increases acetate consumption and decreases extracellular pro-aging factors. Biochim Biophys Acta 1833: 593-601, 2013.

12. Huffman DM, Grizzle WE, Bamman MM, Kim JS, Eltoum IA, Elgavish A and Nagy TR: SIRT1 is significantly elevated in mouse and human prostate cancer. Cancer Res 67: 6612-6618, 2007.

13. Bradbury CA, Khanim FL, Hayden R, Bunce CM, White DA, Drayson MT, Craddock C and Turner BM: Histone deacetylases in acute myeloid leukaemia show a distinctive pattern of expression that changes selectively in response to deacetylase inhibitors. Leukemia 19: 1751-1759, 2005 
14. Hida Y, Kubo Y, Murao K and Arase S: Strong expression of a longevity-related protein, SIRT1, in Bowen's disease. Arch Dermatol Res 299: 103-106, 2007.

15. Stünkel W, Peh BK, Tan YC, Nayagam VM, Wang X, Salto-Tellez M, Ni B, Entzeroth $M$ and Wood J: Function of the SIRT1 protein deacetylase in cancer. Biotechnol J 2: 1360-1368, 2007.

16. Bass P, Carr N and Du Boulay C: Pathology: A Core Text of Basic Pathological Processes with Self-Assessment. 2nd edition. Churchill Livingstone, Edinburgh, 2004.

17. Yang J, Guo R, Kang A, Chen X, Su B, Huang X, Jin Y and $\mathrm{Li}$ Z: A novel histological typing and grading-scale system of colorectal cancer. Nan Fang Yi Ke Da Xue Xue Bao 34: 169-173, 2014 (In Chinese).

18. Dukes CE: The classification of cancer of the rectum. J Pathol 35 : 323-332, 1932.

19. Chueca E, Lanas A and Piazuelo E: Role of gastrin-peptides in Barrett's and colorectal carcinogenesis. World J Gastroenterol 18: 6560-6570, 2012.

20. Fadri-Moskwik M, Weiderhold KN, Deeraksa A, Chuang C, Pan J, Lin SH and Yu-Lee LY: Aurora B is regulated by acetylation/deacetylation during mitosis in prostate cancer cells. FASEB J 26: 4057-4067, 2012

21. Kim SJ, Ao Z, Warnock G and McIntosh CH: Incretin-stimulated interaction between $\beta$-cell Kv1.5 and $\operatorname{Kv} \beta 2$ channel proteins involves acetylation/deacetylation by CBP/SirT1. Biochem J 451: 227-234, 2013

22. Hishida T, Nozaki Y, Nakachi Y, Mizuno Y, Iseki H, Katano M, Kamon M, Hirasaki M, Nishimoto M, Okazaki Y and Okuda A: Sirt1, p53, and p38(MAPK) are crucial regulators of detrimental phenotypes of embryonic stem cells with Max expression ablation. Stem Cells 30: 1634-1644, 2012.

23. Muller PA and Vousden KH: p53 mutations in cancer. Nat Cell Biol 15: 2-8, 2013.

24. Castro RE,Ferreira DM, A fonso MB, Borralho PM, Machado MV Cortez-Pinto $\mathrm{H}$ and Rodrigues CM: miR-34a/SIRT1/p53 is suppressed by ursodeoxycholic acid in the rat liver and activated by disease severity in human non-alcoholic fatty liver disease. J Hepatol 58: 119-25, 2013.

25. Zannini L, Buscemi G, Kim JE, Fontanella E and Delia D: DBC1 phosphorylation by ATM/ATR inhibits SIRT1 deacetylase in response to DNA damage. J Mol Cell Biol 4: 294-303, 2012.

26. Liu L, Wang P, Liu X, He D, Liang C and Yu Y: Exogenous $\mathrm{NAD}(+)$ supplementation protects $\mathrm{H} 9 \mathrm{c} 2$ cardiac myoblasts against hypoxia/reoxygenation injury via Sirt1-p53 pathway. Fundam Clin Pharmacol 28: 180-189, 2014.
27. Marfe G, De Martino L, Tafani M, Irno-Consalvo M,Pasolini MP, Navas L, Papparella S, Gambacurta A and Paciello O: A multicancer-like syndrome in a dog characterized by p53 and cell cycle-checkpoint kinase 2 (CHK2) mutations and sirtuin gene (SIRT1) down-regulation. Res Vet Sci 93: 240-245, 2012.

28. Lee CW, Wong LL, Tse EY, Liu HF, Leong VY, Lee JM, Hardie DG, Ng IO and Ching YP: AMPK promotes p53 acetylation via phosphorylation and inactivation of SIRT1 in liver cancer cells. Cancer Res 72: 4394-4404, 2012.

29. Kriegl L, Vieth M, Kirchner T and Menssen A: Up-regulation of c-MYC and SIRT1 expression correlates with malignant transformation in the serrated route to colorectal cancer. Oncotarget 3: 1182-1193, 2012.

30. Schweiger MR, Hussong M, Röhr C and Lehrach H: Genomics and epigenomics of colorectal cancer. Wiley Interdiscip Rev Syst Biol Med 5: 205-219, 2013.

31. Chong ZZ, Shang YC, Wang S and Maiese K: SIRT1: New avenues of discovery for disorders of oxidative stress. Expert Opin Ther Targets 16: 167-178, 2012.

32. Hubbard BP, Gomes AP, Dai H, Li J, Case AW, Considine T, Riera TV, Lee JE, E SY, Lamming DW, et al: Evidence for a common mechanism of SIRT1 regulation by allosteric activators. Science 339: 1216-1219, 2013.

33. Yao X, Zhao G, Yang H, Hong X, Bie L and Liu G: Overexpression of high-mobility group box 1 correlates with tumor progression and poor prognosis in human colorectal carcinoma. J Cancer Res Clin Oncol 136: 677-684, 2010.

34. Firestein R, Blander G, Michan S, Oberdoerffer P, Ogino S, Campbell J, Bhimavarapu A, Luikenhuis S, de Cabo R, Fuchs C, et al: The SIRT1 deacetylase suppresses intestinal tumorigenesis and colon cancer growth. PLoS One 3: e2020, 2008.

35. Yeung F, Hoberg JE, Ramsey CS, Keller MD, Jones DR, Frye RA and Mayo MW: Modulation of NF-kappaB-dependent transcription and cell survival by the SIRT1 deacetylase. EMBO J 23: 2369-2380, 2004

36. Leko V, Park GJ, Lao U, Simon JA and Bedalov A: Enterocyte-specific inactivation of SIRT1 reduces tumor load in the APC (+/min) mouse model. PLoS One 8: e66283, 2013.

37. Howitz KT, Bitterman KJ, Cohen HY, Lamming DW, Lavu S, Wood JG, Zipkin RE, Chung P, Kisielewski A, Zhang LL, et al: Small molecule activators of sirtuins extend Saccharomyces cerevisiae lifespan. Nature 425: 191-196, 2003.

38. Simic P, Zainabadi K, Bell E, Sykes DB, Saez B, Lotinun S, Baron R, Scadden D, Schipani E and Guarente L: SIRT1 regulates differentiation of mesenchymal stem cells by deacetylating $\beta$-catenin. EMBO Mol Med 5: 430-440, 2013. 